\title{
Sound Field Control in the Automotive Environment
}

Jordan Cheer, Stephen J. Elliott and Woomin Jung 


\section{Introduction}

Sound field control involves the generation and manipulation of acoustic fields using electroacoustic transducers [1]. This encompasses spatial audio reproduction [2], zonal sound control [3], active absorption or reflection of sound [4], and active noise control [5]. In all of these technologies either loudspeakers or structural-acoustic actuators are used to produce a sound field with a controlled spatial and temporal distribution. For example, in the context of spatial audio an array of loudspeakers may be driven to reconstruct a pre-defined sound field, such as that experienced in a concert hall during an orchestral performance [6].

In the automotive environment, sound field control has been used to provide additional functionality and to overcome traditional performance limitations in both the audio system and in the control of vehicular noise. For example, in the context of the car audio system, sound field control methods have been used to improve the typical stereo imaging problems caused by off centre listening [7] and to provide spatial audio reproduction capabilities $[8,9]$. In the context of the noise, vibration and harshness (NVH) performance, active noise control has been employed to control both engine and road noise [10].

The application of active noise control to the automotive environment has been investigated within the automotive industry for in excess of 20 years [10]. This work has generally focussed on reducing the interior engine [11] or road noise [12] in the vehicle cabin using a number of loudspeakers. This process is based on generating a sound field that is out of phase with the unwanted noise produced by the vehicle, and thus reducing it through destructive interference. The performance of active noise control systems is thus dependent on both the spatial and temporal accuracy with which the sound field produced by the control loudspeakers matches the unwanted, disturbance sound field [4].

In the context of the automotive audio system, sound field control has recently been employed to generate multiple independent listening zones in the car cabin [13]. This system allows the occupants of the car cabin to listen to individualised audio entertainment without the need for headphones. This system essentially relies on the same physical principles as active noise control, however, in this case the unwanted noise in each listening zone which must be cancelled is due to the audio content being reproduced in the other listening zones in the car cabin.

This paper presents a review of the limitations of sound field control within the automotive environment. In Section 2 the limitations of global active control in the automotive environment are first outlined, and then practical examples are used to highlight the limitations for both engine and road noise control. In Section 3 it is then shown how some 
of these limitations may be overcome by using a local active control strategy and, once again, a practical automotive application is used to demonstrate the potential advantages of a local control strategy. In Section 4 the physical limitations of active noise control will then be linked to the limitations on the generation of localised audio sound zones within the car cabin and, once again, this will be achieved using the results from a practical sound zone generation system.

\section{Global Active Noise Control}

Global active control of noise in an enclosure, such as a car cabin, aims to minimise the sound pressure level throughout the enclosure. This is usually achieved in theory by minimising the total acoustic potential energy within the enclosure, although in practice this has to be approximated by minimising the sum of the squared pressures measured at a number of error microphone locations. If the control system is implemented as a feedforward controller, as shown in Fig. 1, and consists of $L$ error microphones located in the enclosure, $M$ control loudspeakers and $K$ reference signals, the vector of error signals, e, at a single frequency can be expressed as

$$
\mathbf{e}=\mathbf{d}+\mathbf{G u}=\mathbf{d}+\mathbf{G W x}
$$

where $\mathbf{d}$ is the vector of disturbance signals measured at the error microphones without control, $\mathbf{G}$ is the $(L \times M)$ matrix of transfer responses between the inputs to the control loudspeakers and the pressures measured at the error microphones, $\mathbf{u}$ is the vector of $M$ control signals which drive the control loudspeakers, $\mathbf{x}$ is the vector of reference signals and $\mathbf{W}$ is the control filter matrix. In general, global active control aims to minimise the sum of the squared error signals, which will approximate the total acoustic potential energy in the enclosure provided that a sufficient number of microphones are employed. The cost function that should be minimised in this case is given at a single frequency as

$$
J=\mathbf{e}^{H} \mathbf{e}=\mathbf{u}^{H} \mathbf{G}^{H} \mathbf{G u}+\mathbf{u}^{H} \mathbf{G}^{H} \mathbf{d}+\mathbf{d}^{H} \mathbf{G u}+\mathbf{d}^{H} \mathbf{d} .
$$

The optimal vector of control signals which minimises this cost function is then given in the overdetermined case by [5]

$$
\mathbf{u}_{\text {opt }}=-\left[\mathbf{G}^{H} \mathbf{G}\right]^{-1} \mathbf{G}^{H} \mathbf{d} .
$$

It can be seen from eq. (3) that this optimal solution to the multi-input multi-

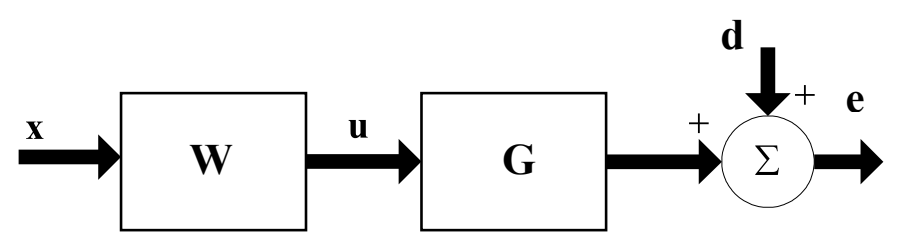

Figure 1 - Multichannel feedforward controller. 
output active noise control problem assumes that disturbance signals are known in advance. In the automotive environment this is unlikely to be the case and instead it is necessary to implement an adaptive algorithm, which iteratively converges towards the optimal solution given by eq. (3). For example, this can be achieved using the filtered- $x$ LMS algorithm [14]. However, eq. (3) is useful to determine the physical limitations on active noise control in the automotive environment without considering the additional complications due to the use of adaptive algorithms.

In order to understand the physical limitations on global active noise control, the interior automotive noise control problem will be represented by the control of the noise produced by a single primary acoustic source located in a rigid walled rectangular enclosure with similar dimensions to a small car interior. Fig. 2 shows the total acoustic potential energy in the rectangular enclosure before control (thick black line) and after control using either a single control source located in one corner of the enclosure (red dot-dashed line) or eight control sources located in the corners of the enclosure (blue dashed line). From this plot it can be seen that with a single control source the system is able to achieve significant attenuation of the compliant mode and the first longitudinal enclosure mode at $72 \mathrm{~Hz}$. Control at higher frequencies, however, is rather limited and this is due to the increasing number of acoustic modes that are excited in a three dimensional enclosure as the frequency of excitation is increased. From the blue dashed line in Fig. 2 it can be seen that when 8 control sources located in the corners of the enclosure are used to minimise the acoustic potential energy, global attenuations are achieved up to around $240 \mathrm{~Hz}$. This increase in the control performance is because the control sources are able to couple into, and therefore control more modes of the enclosure. However, the number of control sources required rapidly increases to an impractical level, since the number of acoustic modes in a three-dimensional enclosure approximately increases with the cube of the excitation frequency.

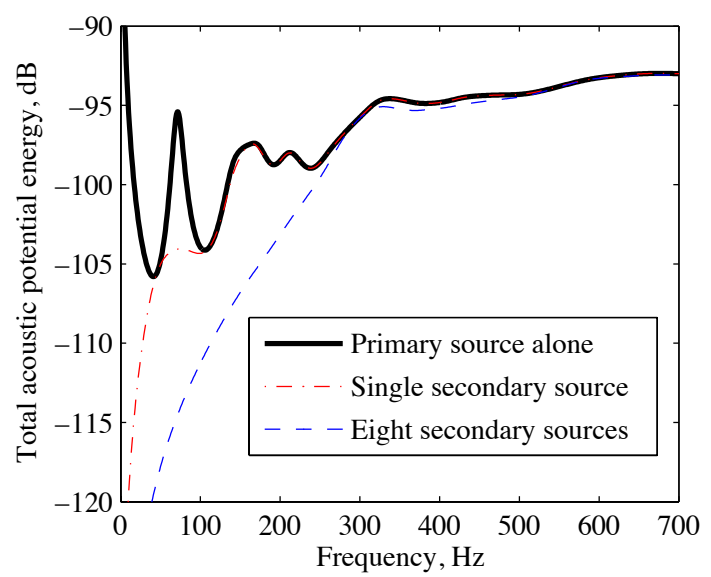

Figure 2 - The total acoustic potential energy in a three-dimensional rectangular enclosure before control (thick black line) and after control using a single secondary control source located in one corner (red dot-dashed line) and eight secondary control sources located in the corners of the enclosure (blue dashed line). 


\subsection{Active Control of Engine Noise}

Feedforward control of engine noise was first demonstrated in the late 1980s [11] and has since been used in various configurations to control the increase in noise level due to lightweight vehicle design [10], to reduce the variation in the engine noise characteristic due to the use of economical engine designs such as variable displacement [15], and to improve the perceived sound quality of the engine noise [16]. A cost effective feedforward engine noise control system can be implemented using an engine speed reference sensor, low-cost microphone error sensors, the car audio loudspeakers and their amplifiers as control sources and the car audio digital signal processing (DSP) capabilities. Such active engine noise control systems have been implemented by a number of manufacturers.

In order to demonstrate the performance limitations of an active engine noise control system, Fig. 3 shows the attenuation achieved by a practical system implementation applied to a small city car with a 2-cylinder engine [17]. The control system in this case employs 8 error microphones mounted on the seat headrests, the four low-frequency car audio loudspeakers as control sources, a reference signal obtained from a tachometer and a filtered- $x$ LMS adaptation algorithm. The results shown in Figure 3 show the attenuation in the sum of the squared pressures measured at 16 microphone locations at the frequency of the first engine order during an engine run-up. From these results it can be seen that, in general, the level of control decreases with increasing frequency and, as shown in the previous section, this is due to the increasing number of acoustic modes excited in the car cabin.

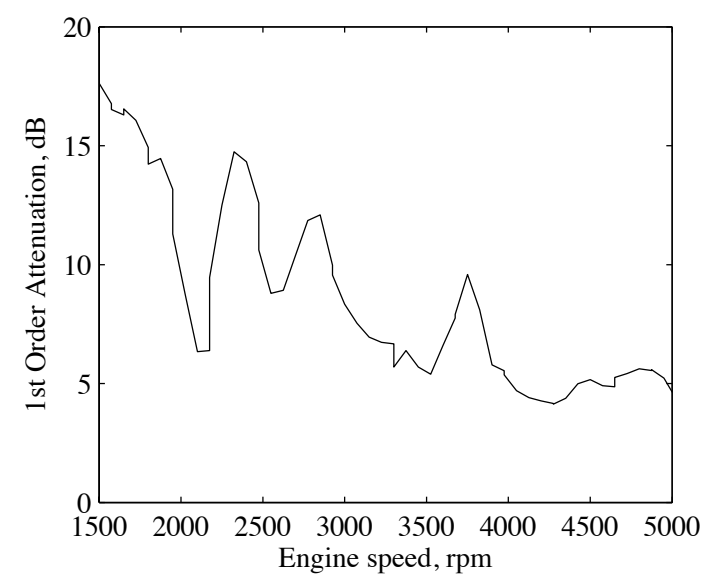

Figure 3 - The attenuation in the sum of the squared pressures measured at 16 microphone locations at the first engine order during an engine run-up. 


\subsection{Active Control of Road Noise}

Although engine noise control has seen a number of commercial implementations, road noise control has been less commercially successful. This is due to the higher costs and challenges of implementation. However, active road noise control is an important technology to allow lightweight vehicle design, which tends to result in an increase in the low frequency broadband interior noise due to the interaction of the tires and the road. Feedforward active noise control systems have previously been developed to reduce the noise levels in the car cabin using reference signals obtained by direct measurement of the vibration due to road excitation $[12,18,19]$. Although these feedforward road noise control systems could again be implemented using low-cost microphone error sensors, the car audio loudspeakers as control sources and the car audio DSP capabilities, it is also necessary to employ at least six accelerometers mounted to the vehicle's suspension and bodywork to obtain reference signals with sufficient coherence with the error signals in order to obtain reasonable levels of control $[12,19]$.

In order to overcome the high cost associated with employing a number of accelerometer reference signals, road noise control systems employing only microphone sensors have been investigated $[20,21]$. One approach in this case is to employ headrest mounted error microphones in a feedback control configuration [20]. However, a higher level of performance has been achieved by employing both headrest mounted error microphones and floor mounted microphones to provide a time-advanced signal to the controller, which can be implemented using either a feedforward or internal model control feedback structure [21]. Fig. 4 shows the performance of this road noise control system implemented in a small city car using the four low-frequency car audio loudspeakers, four headrest error microphones and four additional microphone sensors mounted on the floor of the cabin. Fig. 4 shows the sum of the squared pressures measured at the headrest error microphones and it can be seen that the broadband peak in the road noise between 80 and $200 \mathrm{~Hz}$ has been attenuated to a similar level by both the feedforward and feedback controller implementations and a maximum attenuation of $8 \mathrm{~dB}$ has been achieved at $115 \mathrm{~Hz}$. At higher frequencies it can be seen from these results that the performance of the road noise controller is again rather limited and this can again be linked to the increase in the number of acoustic enclosure modes that are being excited. However, in the feedforward controller architecture there is also a limitation on the control bandwidth due to the low multiple-coherence between the floor microphone signals and the headrest microphone signals at higher frequencies. And, in the case of the feedback implementation, there is a bandwidth limitation due to the delay in the response between the control loudspeakers and the headrest error microphones. 


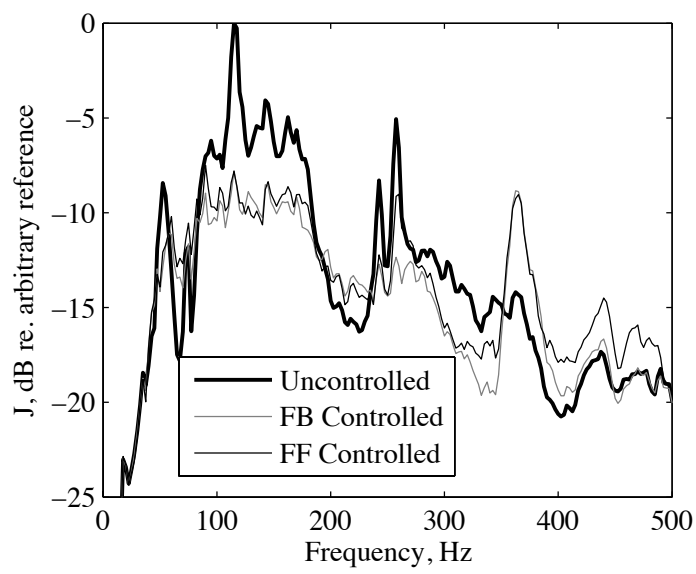

Figure 4 - The sum of the squared pressures measured by the headrest error microphones while the small city car is driven at $50 \mathrm{~km} / \mathrm{h}$ on a pave road surface. The lines show the results before control (thick black line), after control using the optimised feedforward (thin black line) and feedback (thin light line) controllers. The results are plotted in decibels with respect to an arbitrary reference level.

\section{Local Active Noise Control}

In the previous section it has been shown that the bandwidth of global active control in a car cabin sized enclosure is physically limited by the rapid increase in the number of acoustic modes with increasing frequency. Additionally, it has been highlighted that, although a practical feedback road noise control system overcomes some limitations of feedforward implementations in terms of coherence between reference and error signals, it is limited by the delay in the plant response. In order to attempt to overcome these limitations and extend the high frequency limit of active noise control, there has been interest in using local active noise control to create zones of quiet using loudspeakers in the headrest, for example $[22,23]$. The performance of such a local control system depends on the local geometry of the head and headrest [24], but also on the spatial properties of the primary sound field $[25,26]$. Previous work has conducted investigations of the effect of these properties on local active control through calculating the performance for multiple realisations of the primary sound field and averaging the results $[24,27$, 28], however, recently a closed-form solution for the expectation of the controlled sound field has been presented [25]. In the following section this formulation will be used to demonstrate the influence of the primary sound field on the performance of a simple local active control system. Subsequently, some experiments are described in a car, in which the spatial properties of the road noise were measured at an array of microphones. This data is then used to predict the levels of active control that can be achieved with two loudspeakers mounted in the headrest. 


\subsection{Freefield Simulations of Local Active Control}

In order to investigate the effects of the spatial properties of the primary sound field on a local active control system using the formulation presented in [25], we initially consider the case of a single-input single-output control system located in the freefield. The control system consists of a single control source located at $(L, 0)$ and an error sensor located at $(0,0)$, as shown in Fig. 5. The influence of the spatial distribution of the primary field on the shape of the $10 \mathrm{~dB}$ zone of quiet is illustrated in Fig. 5 at a normalized excitation frequency of $k L$ equal to 0.5 , where $k$ is the wavenumber. The shape of the zone of quiet for the simulations of the diffuse sound field, which was achieved with 408 uncorrelated sources forming a sphere in the far field, is the same as that in [27] where the results have been calculated by averaging over multiple random primary sound fields. Also shown in this figure is the shape of this zone when only 21 uncorrelated primary sources are operating, either above or to the right or to the left of the quiet zone. The zone of quiet is greatest when the primary field is mainly from above, since in this case the primary pressure field is almost uniform in the plane shown in Fig. 5, so that reductions at the control point will result in similar reductions at all positions which are a similar distance from the secondary source. When the primary field is mainly from the right, the zone of quiet is somewhat broader in the $x$-direction than that achieved with a diffuse primary field, since the phase variation of the primary field then more nearly matches that of the secondary field in this direction. Conversely, when the primary field is mainly from the left, the phase variations of the primary and secondary field match more accurately in the $y$-direction, and so the extent of the quiet zone has been extended in this direction.

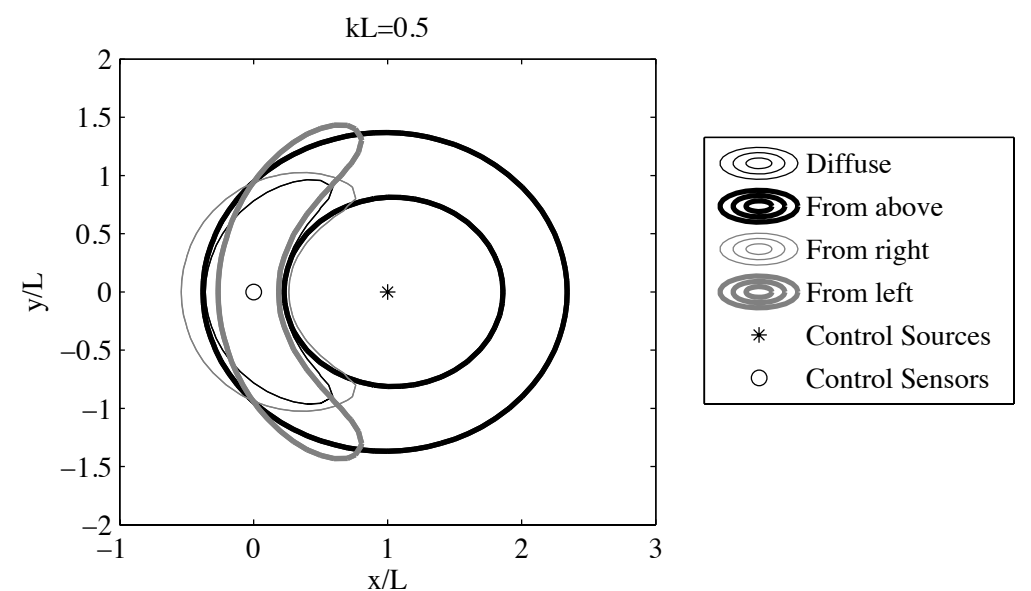

Figure 5 - The extent of the $10 \mathrm{~dB}$ zone of quiet, for a SISO local control system at a normalized excitation frequency of $\mathrm{kL}=0.5$, when the simulation field is diffuse (thin solid black line), mainly coming from above (thick solid black line), mainly from the right hand side (thin dash grey line), and mainly from the left hand side (thick solid grey line). 


\subsection{Local Active Control of Road Noise in a Car}

To evaluate the potential performance of a local active noise control system in an automotive environment, a series of measurements have been conducted in a Ford S-Max $[26,29]$. The experimental setup consisted of an array of 25 microphones mounted on a 0.4 by $0.4 \mathrm{~m}$ grid positioned in front of the headrest on the front passenger seat and two control loudspeakers mounted in the headrest, as shown in Figure 6. The microphone array comprised 12 microphones in an upper grid and 13 in a lower grid, which were separated by $75 \mathrm{~mm}$. Initially, the transfer responses between the voltage inputs to the control loudspeakers and the pressures measured at the microphone grid positions have been measured whilst the vehicle was stationary. The pressures were then measured at the microphones when the vehicle was driven at a variety of speeds on different road surfaces [29].
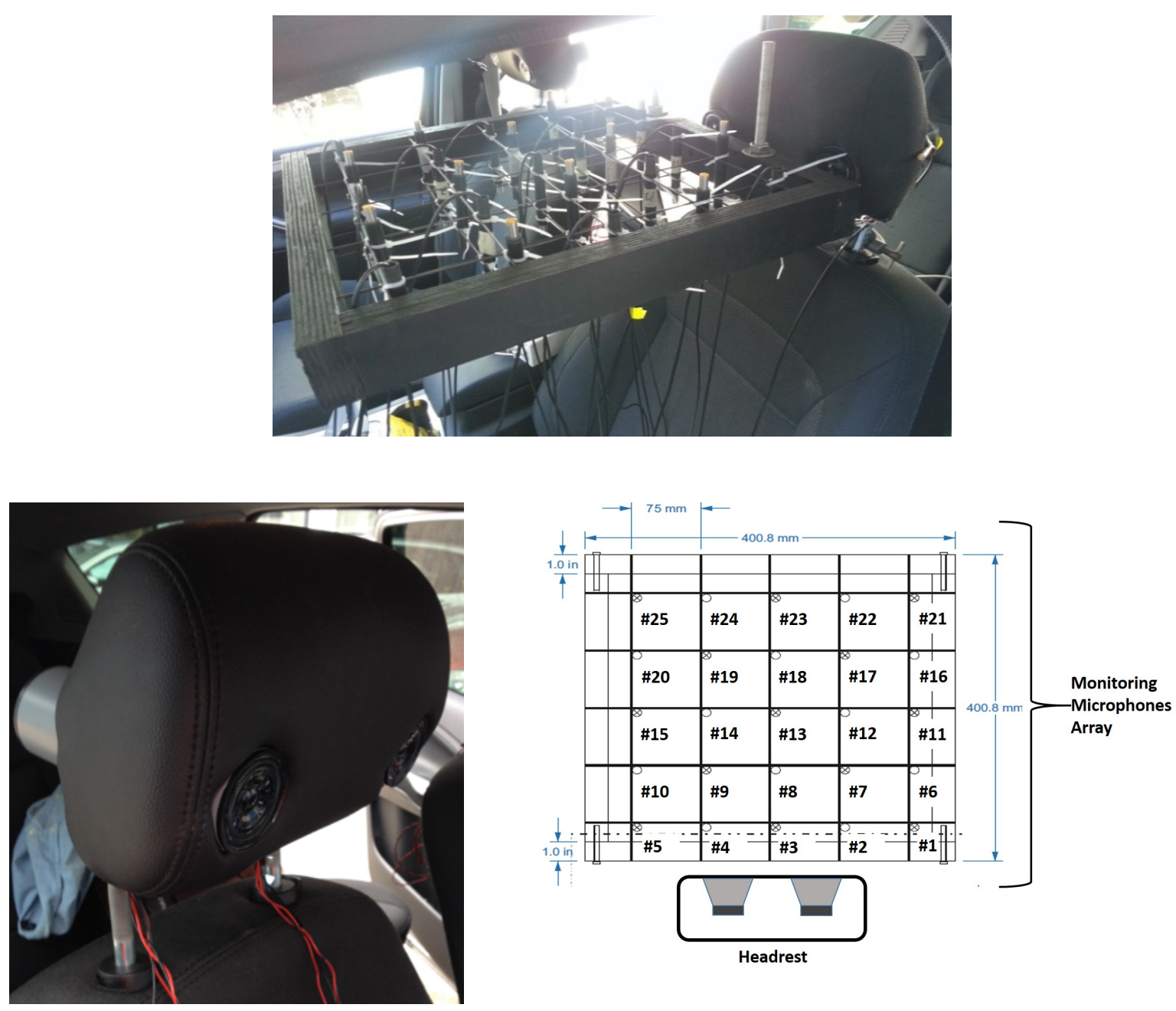

Figure 6 - The geometry of the microphone array, its installation on the front passenger seat and the headrest containing the two control loudspeakers. 
Using the in-car measurements the active control performance has been calculated using the theory presented in [25] and assuming perfect reference signals. Figure 7 shows the performance of the local control system when the car was driven at $50 \mathrm{mph}$ on a relatively smooth road and the two control loudspeakers have been used to minimize the pressures measured at four microphones in the second row of the microphone array, which approximately correspond to locations of the occupant's ears. The black line in Figure 7 shows the A-weight power spectrum averaged over all 25 microphones before control and the red dot-dashed line shows the result of control. From these results it can be seen that attenuation over the full microphone array is achieved up to around $300 \mathrm{~Hz}$, which is an increase from the typical $200 \mathrm{~Hz}$ limit for global active control in a car. However, also shown in Figure 7 is the average A-weighted power spectral density at the 4 error microphones located close to the expected ear positions of the occupant before and after control. In this case it can be seen that significant attenuation is achieved up to $500 \mathrm{~Hz}$ and this clearly demonstrates the potential performance increase that may be achieved by focusing the active control at the occupant's ears.

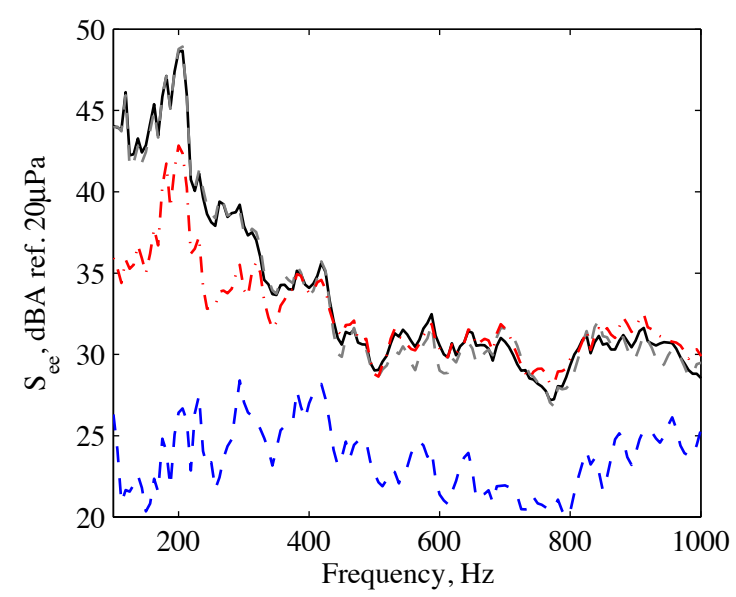

Figure 7 - The A-weighted averaged power spectral density before and after control of road noise using the local control system with two headrest loudspeakers and 4 error microphones for a $50 \mathrm{mph}$ smooth road driving condition. No control averaged over 25 mics (black line); control averaged over 25 mics (red dot-dashed line); no control averaged over 4 mics (grey dashed line); control averaged over 4 mics (blue dashed line).

\section{Sound Field Control for Audio Zone Generation}

In addition to the use of sound field control to attenuate unwanted automotive noise, it can also be used in the automotive environment to enhance the functionality of the audio system, as discussed in the introduction. One particular area of interest is the generation of independent listening zones in the car cabin. This problem has been approached from two principle directions including directional loudspeakers $[30,31]$ and sound field control $[13,32,33]$. A commercial system has recently been presented which relies on both of these operating principles [34], however, these ideas were first 
presented in [13]. In the following section a practical sound zone control system is described, which utilises both active sound control principles, based on controlled constructive and destructive interference between sources, and directional loudspeakers. The performance of this system is assessed in terms of the generation of independent listening zones in the car cabin and the physical limitations are discussed.

\subsection{Practical Sound Zone Control System}

The generation of independent listening zones in a car cabin would ideally be achieved using the standard car audio loudspeakers. However, similarly to the limits on achieving active control presented in the previous sections, it has been shown that using the standard car audio loudspeakers can only achieve separation between listening zones at low frequencies $[13,32]$. Therefore, similarly to the implementation of local active noise control systems, the standard car audio system must be supplemented with loudspeakers mounted in closer proximity to the desired listening zones. In the presented practical example the car cabin personal audio system installed in a Ford S-Max consists of the four standard low-frequency car audio loudspeakers and an additional array of eight phase-shift loudspeakers, with one loudspeaker mounted on each side of each headrest, as shown in Fig. 8. The individual phase-shift loudspeakers have been designed to achieve a hypercardioid directivity, as detailed in [35].

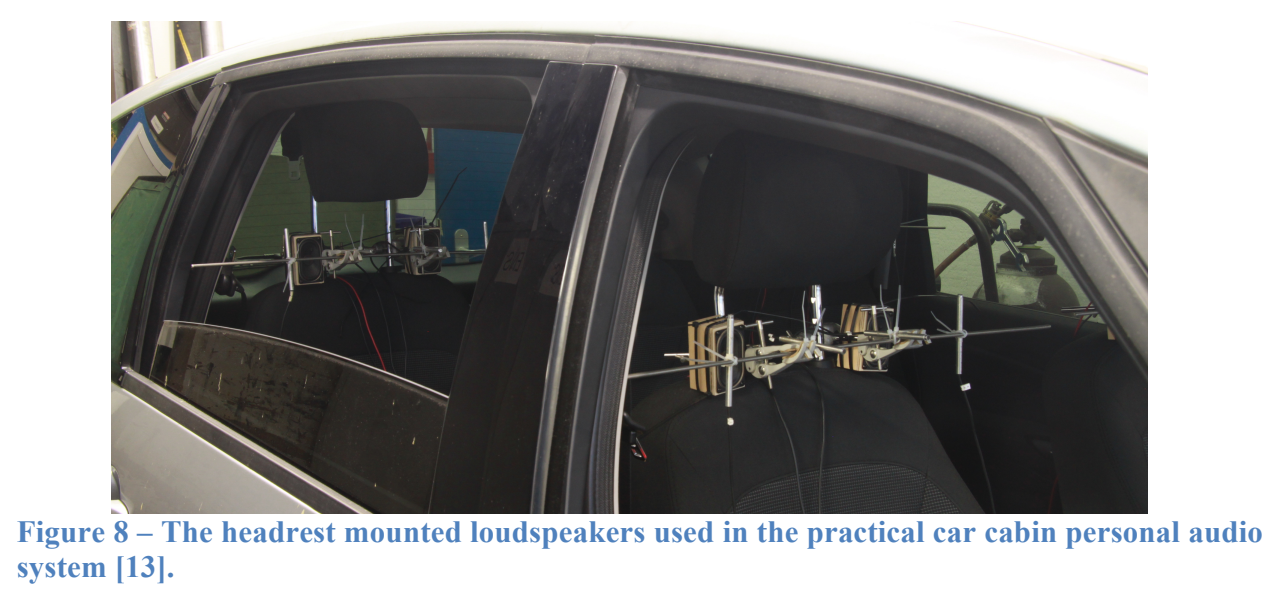

In addition to the design of the physical loudspeaker array, the implementation of personal audio systems requires the optimisation of the signals driving the loudspeakers. In practice this requires a bank of filters to be designed which adjust the magnitude and phase of the audio signals being sent to the loudspeakers. A number of different methods of implementing this filter optimisation process have been proposed [36-38], however, here we will use the least squares design method due to its ability to produce high quality audio $[13,37]$. 
The performance of personal sound zone systems is generally characterised by the difference in reproduced sound level between the independent listening zones. In the considered car cabin application the system aims to produce one listening zone in the front seats and one listening zone in the rear seats. The ability of the sound zone system to produce these independent listening zones can be assessed using the acoustic contrast [36], which is given by the ratio between the sum of the squared pressures measured in the listening zone to the sum of the squared pressures measured in the quiet zone. Fig. 9 shows the acoustic contrast for the implemented car cabin sound zone control system when a listening zone is produced in the front seats Fig. 9a and in the rear seats Fig. 9b. The performance is shown for the standard car audio loudspeakers (thin lines) and for the headrest loudspeaker array (thick lines) when it is measured at 4 microphones at each headrest. From these results it can be seen that in both configurations the car audio loudspeakers are able to achieve significant acoustic contrast up to around $200 \mathrm{~Hz}$, which is similar to the limit on global active control in the automotive environment discussed in Section 2. At higher frequencies the car audio loudspeakers are not able to achieve significant separation between the two listening zones, due to the rapid increase in the number of acoustic modes excited within the enclosure. At higher frequencies it can be seen from Fig. 9 that the headrest loudspeaker arrays are able to improve the acoustic contrast, although there are some limitations when generating a rear listening zone due to the fixed directivity of the individual headrest loudspeakers and there orientations relative to the listening zones [13]. Nevertheless, the increase in the performance bandwidth is comparable to that achieved in the context of local active noise control, as discussed in Section 3.

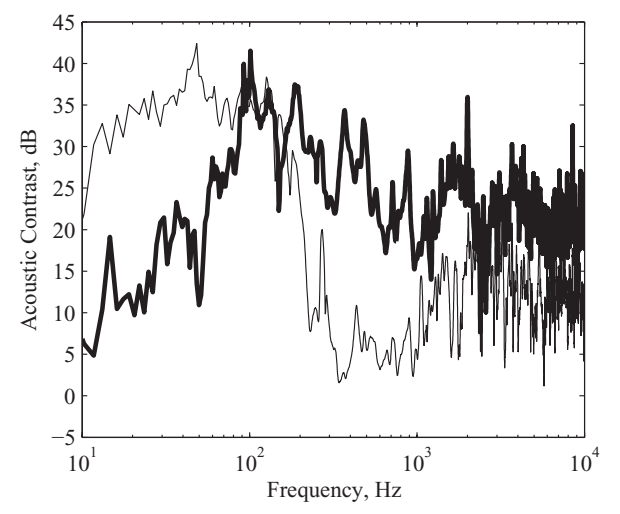

(a) Acoustic contrast, front bright zone

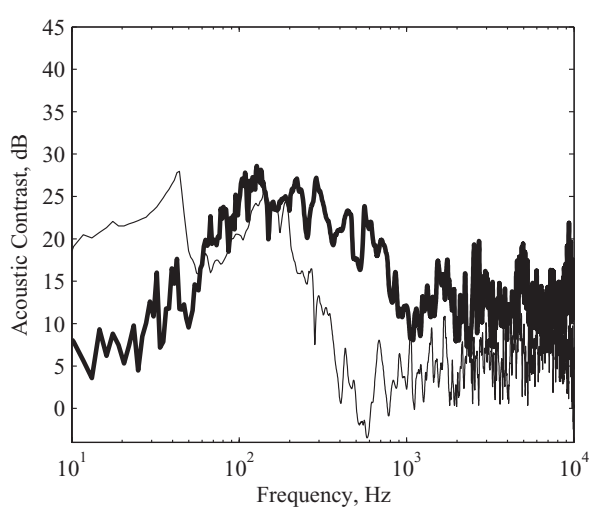

(b) Acoustic contrast, rear bright zone

Figure 9 - The acoustic contrast between the listening and quiet zones achieved by the car cabin sound zone control system when producing (a) a listening zone in the front of the car and (b) when producing a listening zone in the rear of the car. The thick lines show the performance achieved by the headrest loudspeaker array and the thin lines show the performance achieved by the standard car audio loudspeakers. 


\section{Conclusions}

The use of sound field control in the automotive environment offers the potential to improve the acoustic experience of the vehicle's occupants. This can include both refinement of the vehicle NVH through active noise control and additional functionality in the car audio system. This paper has presented a review of the limitations of sound field control within the automotive environment in the context of both active control of engine and road noise, and the generation of independent audio listening zones. In both of these areas, sound field control using the standard car audio loudspeakers is limited to frequencies below around $200 \mathrm{~Hz}$ due to the rapid increase in the number of acoustic modes with frequency. In both applications of sound field control it is necessary to resort to local control systems, with the control loudspeakers located in close proximity to the listening positions, in order increase the upper frequency at which control can be achieved.

\section{References}

[1] F. Rumsey, Sound Field Control, J. Audio Eng. Soc., 61(12), December 2013.

[2] F. Rumsey, Spatial Audio, Focal Press, Oxford, 2001.

[3] T. Betlehem, W. Zhang; M.A. Poletti, T.D. Abhayapala, Personal Sound Zones: Delivering interface-free audio to multiple listeners, IEEE Signal Process. Magazine, 32(2), pp.81-91, 2015.

[4] H. Zhu, R. Rajamani, and K. A. Stelson. Active control of acoustic reflection, absorption, and transmission using thin panel speakers. J. Acoust. Soc. A., 113(2), pp.852-870, 2003.

[5] P.A. Nelson and S.J. Elliott, Active control of sound, Academic Press, 1991.

[6] F. Fazi, P. Nelson, J.E. Christensen and J. Seo, Surround system based on threedimensional sound field reconstruction. In Proc. of Audio Eng. Soc. Convention $125,2008$.

[7] M.J. Smithers, Improved stereo imaging in automobiles, in Proc. of Audio Eng. Society Convention 123, 2007.

[8] M.R. Bai, and C.-C. Lee, Comparative study of design and implementation strategies of automotive virtual surround audio systems. J. Audio Eng. Soc., 58(3), pp.141-159, 2010.

[9] S. Brix, C. Sladeczek, A. Franck, A. Zhykhar, C. Clausen and P. Gleim, Wave field synthesis based concept car for high-quality automotive sound. In Proc. of $48^{\text {th }}$ Audio Eng. Soc. Conf., 2012. 
[10] S.J. Elliott, Active noise and vibration control in vehicles, in: X. Wang (Ed.), Vehicle Noise and Vibration Refinement, Woodhead Publishing, Cambridge, 2010, pp. 235-251.

[11] S.J. Elliott, I.M. Stothers, P. Nelson, M.A. McDonald, D.C. Quinn, T.J. Saunders, The active control of engine noise inside cars, in Proc. of Inter-noise 88, 2, pp. 987-990, 1988.

[12] T.J. Sutton, S.J. Elliott, M.A. McDonald, T.J. Saunders, Active control of road noise inside vehicles, J. Noise Control Eng., 42, pp.137-146, 1994.

[13] J. Cheer S.J. Elliott and M.F. Simón Gálvez, Design and implementation of a car cabin personal audio system, J. Audio Eng. Soc., 62(6), pp. 412-424, 2013.

[14] S.J. Elliott, I. Stothers, and Philip A. Nelson. A multiple error LMS algorithm and its application to the active control of sound and vibration, IEEE Trans. Acoust., Speech, Signal Process., 35(10), pp.1423-1434, 1987.

[15] R. Schirmacher, R. Kunkel, M. Burghardt, Active Noise Control for the 4.0 TFSI with Cylinder on Demand Technology in Audi's S-Series, Society of Automotive Engineers, Graz, no. 2012-01-1533, 2012.

[16] Y. Kobayashi, T. Inoue, H. Sano, A. Takahashi, K. Sakamoto, Active sound control in automobiles, in Proc. of the Inter-noise, 2008.

[17] J. Cheer, Active control of the acoustic environment in an automobile cabin, $\mathrm{PhD}$ Thesis, University of Southampton, U.K., 2012.

[18] R. Bernhard, Active control of road noise inside automobiles, in Proc. of ACTIVE 95, pp. 21-32, 1995.

[19] S.-H. Oh, H.-S. Kim, Y. Park, Active control of road booming noise in automotive interiors, J. Acoust. Soc. Am., 111(1), 180-188, 2002.

[20] J. Cheer and S.J. Elliott, The design and performance of feedback controllers for the attenuation of road noise in vehicles, Int. J. Acoust. Vib., 19(3), 2014.

[21] J. Cheer and S.J. Elliott, Multichannel control systems for the attenuation of interior road noise in vehicles, Mech. Sys. Signal Process., 60-61, pp.753-769, 2015.

[22] B. Rafaely, S.J. Elliott, and J. Garcia-Bonito, Broadband performance of an active headrest, J. Acoustic. Soc. Am., 106(2), pp.787-793, 1999.

[23] M. Pawelczyk, Adaptive noise control algorithms for active headrest system, Control Engineering Practice, 12(9), pp.1101-1112, 2004.

[24] J. Garcia-Bonito, S.J. Elliott, and C.C. Boucher,. Generation of zones of quiet using a virtual microphone arrangement. J. Acoust. Soc. Am., 101(6), 3498-3516, 1997.

[25] S.J. Elliott and J. Cheer, Modelling local active sound control with remote sensors in spatially random pressure field, J. Acoust. Soc. Am., 137(4), 2015.

[26] S.J. Elliott, W. Jung and J. Cheer, The spatial properties and local active control of road noise, in Proc. of Euro-noise, 2015. 
[27] P. Joseph, S. J. Elliott, and P. A. Nelson, Near field zones of quiet, J. Sound Vib. 172, 605-627, 1994.

[28] J. Garcia-Bonito and S. J. Elliott, "Local active control of diffracted diffuse sound fields,” J. Acoust. Soc. Am. 98(2), 1017-1024, 1995.

[29] W. Jung, Local active sound control with remote loudspeakers and microphones, Master's thesis, University of Southampton, U.K., 2014.

[30] F. P. Thigpen, "Vehicle audio system with directional sound and reflected audio imaging for creating a personal sound stage." United States Patent, US 2004/0109575 A1, June 2004.

[31] S. Goose and F. Arman, "System and method for creating personalized sound zones.” United States Patent, US 2006/0262935, November 2006.

[32] S. Berthilsson, A. Barkefors, L.-J. Brännmark, M. Sternad, Acoustical Zone Reproduction for Car Interiors Using a MIMO MSE Framework, in Proc. of 48th International Audio Eng. Soc. Conf., 2012.

[33] W.F. Druyvestseyn and J. Garas, Personal Sound, J. Audio Eng. Soc., 45(9), pp.685-701, 1997.

[34] D. Shewchuk, "Harman unleashes new potential for in-car listening experiences and sound management", http://news.harman.com/Press-Releases/HARMANUnleashes-New-Potential-for-In-Car-Listening-Experiences-and-SoundManagement-212.aspx, January 2015, (accessed 06/04/2015).

[35] M.F. Simón Gálvez, Loudspeaker Arrays for Family Television, Master's thesis, University of Southampton, UK, 2011.

[36] J.-W. Choi and Y.-H. Kim, Generation of an Acoustically Bright Zone with an Illuminated Region Using Multiple Sources, J. Acous. Soc. Am., 111, pp.16951700, 2002.

[37] M.F. Simón Gálvez, S. J. Elliott, and J. Cheer, A superdirective array of phase shift sources. J. Acous. Soc. Am., 132(2), pp.746-756, 2012.

[38] P. Coleman, P.J. Jackson, M. Olik, and J.A. Pedersen, Personal audio with a planar bright zone, J. Acoust. Soc. Am., 136(4), 1725-1735, 2014. 